\title{
Transcriptome-wide Identification and Characterization of microRNAs and Their Targets in a Highly Adaptable Conifer Platycladus orientalis
}

\author{
Ying Yang, Xian-Ge Hu, and Bingsong Zheng \\ State Key Laboratory of Subtropical Silviculture, Zhejiang A \& F University, Lin'an, Hangzhou, \\ Zhejiang 311300, China \\ Yue Li \\ Beijing Advanced Innovation Center for Tree Breeding by Molecular Design, National \\ Engineering Laboratory for Tree Breeding, Key Laboratory of Genetics and Breeding in Forest \\ Trees and Ornamental Plants, Ministry of Education, College of Biological Sciences and \\ Technology, Beijing Forestry University, Beijing 100083, China \\ Tongli Wang \\ Department of Forest and Conservation Sciences, Faculty of Forestry, University of British \\ Columbia, Vancouver, BC, Canada V6T $1 Z 4$ \\ Anket Sharma and Huwei Yuan \\ State Key Laboratory of Subtropical Silviculture, Zhejiang A \& F University, Lin'an, Hangzhou, \\ Zhejiang 311300, China \\ Jian-Feng Mao \\ Beijing Advanced Innovation Center for Tree Breeding by Molecular Design, National \\ Engineering Laboratory for Tree Breeding, Key Laboratory of Genetics and Breeding in Forest \\ Trees and Ornamental Plants, Ministry of Education, College of Biological Sciences and \\ Technology, Beijing Forestry University, Beijing 100083, China
}

AdDitional INDEX words. Cupressaceae, miRNA, pathway, response, targets

\begin{abstract}
MicroRNAs (miRNAs) are short noncoding RNAs (20-25 nucleotides) that regulate gene expression posttranscriptionally. However, identification and characterization of miRNAs remain limited for conifer species. In this study, we applied transcriptome-wide miRNAs sequencing to a conifer species Platycladus orientalis, which is highly adaptable to a wide range of environmental adversities, including drought, barren soil, and mild salinity. A total of 17,181,542 raw reads were obtained from the Illumina sequencing platform; 31 conserved and 91 novel miRNAs were identified, and their unique characteristics were further analyzed. Ten randomly selected miRNAs were validated by quantificational realtime polymerase chain reaction. Through miRNA target predictions based on psRNATarget, 2331 unique mRNAs were predicted to be targets of $P$. orientalis miRNAs that involved in 187 metabolic pathways in KEGG database. These targets included not only important transcription factors (e.g., class III homeodomain leucine zipper targeted by por-miR166d) but also indispensable nontranscriptional factor proteins (i.e., por-miR482a-3p regulated nucleotide-binding site leucinerich repeat protein). Interestingly, six miRNAs (por-miR16, -miR44, -miR60-5p, -miR69-3p, -miR166b-5p, and -miR395c) were found in adaptation-related pathways (e.g., drought), indicating their possible involved in this species' stress-tolerance characteristics. The present study provided essential information for understanding the regulatory role of miRNAs in $P$. orientalis and sheds light on their possible use in tree improvement for stress tolerance.
\end{abstract}

miRNAs have been identified in more than 270 species that span the two major kingdoms of life: prokaryotes and eukaryotes (Kozomara et al., 2018). miRNAs are a group of endogenous small ribonucleic acid (sRNA) molecules, generally 20 to 25

Received for publication 27 May 2021. Accepted for publication 21 Sept. 2021. Published online 19 November 2021.

This research was funded by the Scientific Research and Development Fund Project of Zhejiang A \& F University (NO. 2020FR029) and the National Natural Science Foundation of China (NO. 31670664).

Y.Y. and X-G.H. contributed equally to this work.

H.Y. and J-F.M. are the corresponding authors. E-mail: hwyuan@zafu.edu. cn or jianfeng.mao@bjfu.edu.cn.

This is an open access article distributed under the CC BY-NC-ND license (https://creativecommons.org/licenses/by-nc-nd/4.0/). nucleotides (nt) in length, which regulate target gene expression via the RNA interference pathway at translational repression or posttranscriptional degradation stage (Lagos-Quintana et al., 2001). The important miRNA roles have been discovered in regulating metabolism (Vienberg et al., 2017), tissue initiation and development (Xie et al., 2015), signal transduction (Fendler et al., 2011), cell cycle progression (Zheng et al., 2012), immune response (Chen et al., 2013), and response to different abiotic and biotic stress (Ferdous et al., 2015; Sun, 2012) of plants and animals. The pivotal roles of plant miRNAs have been demonstrated in different biological and metabolic processes including plant development, nutrient homeostasis, antibacterial reactions, phytohormones signaling, and response to stress (Ferdous et al., 2015; Liu and El-Kassaby, 2017; Qiu et al., 2016; Xie et al., 2015). 
In particular, a growing body of evidence suggests that miRNAs are involved in the responses to different stresses to conifers and other gymnosperms (Li et al., 2017; Liu and El-Kassaby, 2017). miRNAs generally bind to the $3^{\prime}$-UTR (untranslated region) of their corresponding target mRNAs and restrain protein production by destabilizing the mRNA and translational silencing and thus regulate the expression of specific genes. For example, the GRAS family protein and nucleotide-binding site leucine-rich repeat (NBS-LRR) resistance protein gene were key transcription factors for Pinus densata response to environmental stress, and their expression patterns has been experimentally demonstrated to be affected by miR171 and miR482, respectively (Qiu et al., 2016).

To date, there are no reports on miRNA from Platycladus orientalis (L.) Franco (Cupressaceae), an ecologically important conifer with high adaptability to a wide range of environmental adversities, including drought, barren soil, and mild salinity (Dong et al., 1990; Flora of China Editorial Committee, 1978; $\mathrm{Hu}$ et al., 2015, 2017). However, due to the limited genomic information, the physiological and genetic mechanisms of its adaptability are not yet well understood. Therefore, the identification of $P$. orientalis miRNAs is an important step to understand the regulatory networks in biological and metabolic processes. In general, the plant miRNAs often have been identified by two approaches: 1) experimental sequencing and 2) bioinformatic prediction. Using in silico prediction, miRNAs are identified by homology querying of various genomic resources (e.g., expressed sequence tags, genomic survey sequences, and high-throughput genomic sequences). Recently, the advent of high-throughput sequencing technologies has greatly accelerated the discovery of medium-to-low abundant and species-specific miRNAs from diverse plant species including conifer trees, such as $P$. densata (Wan et al., 2012), Picea balfouriana (Li et al., 2017), and Picea glauca (Liu and El-Kassaby, 2017).

The transcriptome dataset of $P$. orientalis generated by highthroughput sequencing technology has been published, and 148,867 unigenes were generated from terminal buds, microstrobilus, female strobilus, biennial leaves, and cambium tissues $(\mathrm{Hu}$ et al., 2016). This transcriptome dataset provides a useful reference sequence to identify new miRNA in $P$. orientalis, and these potentially discovered miRNAs will improve our understanding of the regulatory networks in this ecologically important species.

The main objective of this study was to identify miRNAs and their potential targets in P. orientalis, as well as a corresponding metabolic pathway involved in stress response. We constructed an independent $P$. orientalis sRNA library by high-throughput Solexa Illumina sequencing. The conserved miRNAs were then identified by the sequences blast in the public miRNA database, and novel miRNAs were identified according to previously published criteria for plant miRNAs (Meyers et al., 2008). Additionally, quantitative reverse transcriptase polymerase chain reaction (qRT-PCR) was used to validate the accuracy and reproducibility of the newly identified $P$. orientalis miRNAs. This study formed a basis for further studying the regulative functions of miRNAs in the development and growth of $P$. orientalis.

\section{Materials and Methods}

Plant materials. During the flowering season (April-May), terminal buds, microstrobilus, female strobilus, biennial leaves, and cambium tissues were sampled from five adult $P$. orientalis trees located in the Beijing Botanical Garden (Beijing, China).
All tissue samples were washing with tap water and deionized water to remove unwanted contamination, immediately frozen in liquid nitrogen, and stored at $-80^{\circ} \mathrm{C}$ until further use.

SMall RNA library PREParation and SEQUeNCING. Total RNA was separately isolated from the tissue samples using RNeasy Plant Mini Kit (Qiagen, Hilden, Germany), according to the manufacturer's protocol. Equal quantities of total RNA from terminal buds, microstrobilus, female strobilus, biennial leaves, and cambium were pooled to generate an sRNA library using the TruSeq Small RNA Sample Prep Kit (Illumina, San Diego, CA). The polyacrylamide gel electrophoresis was used to enrich sRNA molecules in the range of 18 to $30 \mathrm{nt}$, which were ligated to adapters at $5^{\prime}$ and $3^{\prime}$ ends and amplified by PCR. After that, the products were purified and subjected to sequencing on Illumina HiSeqTM 2500 platform (Beijing, China).

Small RNA SEQueNCING AND Data PRoCessing. After removing the adaptor sequences and poor-quality reads, high-quality sequences with 18 to $30 \mathrm{nt}$ were reserved. All unique sequences were searched against the Rfam and National Center of Biotechnology Information (NCBI) noncoding RNA databases using the BLASTN program (Altschul et al., 1990), and ribosomal RNA (rRNA), transfer RNA (tRNA), small nuclear RNA (snRNA), and small nucleolar RNA (snoRNA) as well as those containing poly-A tail were identified and removed from the sRNA library.

The sRNA sequences were then searched against known miRNA sequences in miRBase (Release 22.1), which contains 48,885 miRNA products from 271 species (Kozomara et al., 2018 ), to identify $P$. orientalis conserved miRNAs with less than two mismatches (Griffiths-Jones et al., 2008). The remaining reads that did not match any of the sequences in miRBase were used to predict novel miRNAs by Mireap (Li et al., 2012). Novel miRNAs were identified according to previously established criteria for plant miRNAs (Meyers et al., 2008). Because most of the pre-miRNA sequences are rapidly degraded after endonuclease Dicer-like cleavage, only the mature miRNA was obtained. Therefore, to produce a more comprehensive prediction for the miRNAs, publicly available RNA data of a closely related plant species (Pinus taeda) were also used as a reference to predict the secondary structure of $P$. orientalis miRNAs. If a reference sequence with miRNA-like alignment met the criteria (Meyers et al., 2008), it was considered a putative miRNA. Additionally, the secondary structure of the identified P. orientalis miRNAs will be validated by Mfold (Zuker, 2003).

Characterization of CONSERVEd $\boldsymbol{P}$. orientalis mirnas. Among the group of conserved miRNAs, highly conserved miRNA families share conserved functions in plant growth, development, and stress responses. Therefore, the typically conserved $P$. orientalis miRNA families were selected for phylogenetic analysis to validate the conservation between the newly identified and already discovered members in a same miRNA family. We derived different precursor sequences of miRNAs from the miRBase release 22.1 (Kozomara et al., 2018) and subjected them to multiple alignment with newly identified $P$. orientalis pre-miRNAs using the ClustalW2 program (Larkin et al., 2007). The phylogenetic analysis was performed to determine the evolutionary relationships by the program MEGA5.0 (Tamura et al., 2011), and a phylogenetic tree was constructed using the neighbor-joining (NJ) method with 1000 bootstrap replicates.

Quantitative RT-PCR VAlidation OF PARTIAL CANDidate MIRNAs. To confirm the accuracy and reproducibility of the identified miRNAs, 10 identified miRNAs were randomly selected, 
and the same RNA preparation samples used in sequencing were subjected to qRT-PCR with U6 snRNA as reference (Bustin et al., 2009). Total RNA isolated from the collected plant materials was used for the first strand cDNA synthesis by FastQuant RT Kit (with gDNAase; Tiangen, Beijing, China) following the manufacturer's protocol. The primers for qRT-PCR were listed in Supplemental Table 1. qRT-PCR was performed using a SYBR Premix EX Taq Kit (TaKaRa Bio., Otsu, Japan) based on Applied Biosystems 7300 Sequence Detection System (Life Technology, Foster City, CA). The reaction system in a $20-\mu \mathrm{L}$ reaction volume containing $1 \mu \mathrm{L}$ diluted cDNA, $0.6-\mu \mathrm{L}$ primers, and $10 \mu \mathrm{L} 2 \times$ PCR Master Mix (Tiangen). During the reaction, the fractional cycle number at which the fluorescence signal passes a fixed threshold is defined as threshold cycle (CT), and after the reactions were completed, the CT was automatically recorded. All reactions were performed with three replicates. The $\Delta \mathrm{Ct}\left(\mathrm{CT}_{\text {sample }}-\mathrm{CT}_{\text {ref. }}\right)$ was calculated for analyzing miRNAs expression level (Livak and Schmittgen, 2001).

Prediction of miRna targets and FUnCtional ANNOTATION. The target genes of the new identified miRNAs were predicted using psRNATarget (Dai et al., 2018) with the following parameters: maximum expectation value at 3; target accessibility-allowed energy to unpair target site as $25 ; 17$ bp upstream and $13 \mathrm{bp}$ downstream for the flanking length around target site for target accessibility analysis; the range of central mismatch that will lead to translational inhibition is 10 to $11 \mathrm{nt}$; maximum mismatch at complementary site $\leq 4$ without 0.5 gaps.

The putative functions of the identified miRNA target genes were assigned by performing BLASTX search against the nonredundant database of NCBI with an e-value of 1e-10. To attain a better understanding of the miRNA function and unravel the miRNA gene regulatory network in terms of molecular function, cellular component, and biological process, gene ontology (GO) annotation and enrichment analysis were carried out by webbased BLAST2GO (Conesa et al., 2005). Finally, the metabolic pathways and their networks regulated by the potential miRNAs were identified through the target transcripts BLAST against Kyoto Encyclopedia of Genes and Genomes (KEGG) database.

\section{Results}

DeEP SEQUENCING. A total of 17,181,542 raw reads were obtained from the deep sequencing of the $P$. orientalis sRNA library. After removal of low-quality reads, adaptor sequences, and sequences with length outsides of 18 to $30 \mathrm{nt}, 7,616,082$ clean reads, representing 1,091,289 unique reads, were reserved. The numbers and proportions of different types of sRNA are shown in Table 1. Among the unique clean reads, only $36.77 \%$ could be annotated, suggesting that the sRNAs have not been mined extensively in previous studies and that our study had greater potential to discover more miRNAs.

IDENTIFICATION OF $\boldsymbol{P}$. ORIENTALIS CONSERVED MIRNAS. Thirtyone conserved miRNAs were identified comprising 18 miRNA families, the sequences of mature and precursor, and the predicted hairpin structures, shown in Table 2, Supplemental Table 2, and Supplemental Fig. 1, respectively. The length of $P$. orientalis mature miRNAs varied from 19 to $23 \mathrm{nt}$, of which $21(41.2 \%)$ and 22 nt $(35.3 \%)$ were the two major size classes (Table 2$)$. The length of miRNA precursors ranged from 68 to $132 \mathrm{nt}$ with an average value of 95. The minimal folding free energy (MFE) of $P$. orientalis miRNA precursors varied from -19.2 to $-64.5 \mathrm{kcal} / \mathrm{mol}$
Table 1. Distribution of Platycladus orientalis small ribonucleic acid (sRNA) among different categories.

\begin{tabular}{lrcrrr}
\hline & \multicolumn{2}{c}{ Unique sRNA } & & \multicolumn{2}{c}{ Total sRNA } \\
\cline { 2 - 3 } \cline { 5 - 6 } Category & Number & Proportion (\%) & & Number & Proportion (\%) \\
\hline Total & $1,091,289$ & & & $7,616,082$ & \\
rRNA $^{\mathrm{z}}$ & 383,368 & 35.13 & & $4,829,219$ & 63.41 \\
tRNA $^{\mathrm{y}}$ & 168,03 & 1.54 & & 276,215 & 3.63 \\
snoRNA $^{\mathrm{x}}$ & 1,094 & 0.1 & & 8,693 & 0.11 \\
snRNA $^{\mathrm{w}}$ & 17 & 0 & & 50 & 0 \\
Annotated & 401,282 & 36.77 & & $5,114,177$ & 67.15 \\
\hline
\end{tabular}

${ }^{\mathrm{z}}$ Ribosomal RNA.

y Transfer RNA.

${ }^{\mathrm{x}}$ Small nucleolar RNA.

${ }^{\text {w }}$ Small nuclear RNA.

with an average value of $-43.9 \mathrm{kcal} / \mathrm{mol}$. Additionally, each miRNA member had a different expression abundance from 2 to 8735 , with an average expression value of 729 (Table 2).

In conserved miRNAs of $P$. orientalis, 15 new miRNA members belonging to 10 conserved families were the first reported in plant (Table 2). In addition, six (por-miR159a, -miR166a-5p, -miR166b-5p, -miR319a, -miR393a, and -miR1083a-5p) were further validated by qRT-PCR in the randomly selected miRNA validation experiment. Additionally, most of the identified conserved miRNAs of $P$. orientalis $(81 \%)$ have been found in four other closely related conifer species; in particular, por-miR1314a, -miR159a, and -miR482a-3p were found in more than three conifers. Interestingly, one $P$. orientalis miRNA family may contain diverse members (e.g., the por-MIR166 family has six members), whereas only one member exists in 11 miRNA families (Supplemental Fig. 2).

Phylogenetic analysis of MiR166 And MiR482. Among the conserved $P$. orientalis miRNAs, MIR166 is the most abundant miRNA family with six members and 10,723 expression abundances. This miRNA family is known to be drought-responsive and a regulator in axillary meristem initiation and leaf and vascular development in various plant species (Ferdous et al., 2015; Kantar et al., 2011; Sun, 2012). Additionally, miR482, another important stress-responsive miRNA, was identified; it is ubiquitously distributed in gymnosperm, monocot, and dicot plants and plays a critical regulatory role in disease resistance in plants (Zhu et al., 2013). A new member of miR482 was identified in our study, and its target was predicted in NBS-LRR proteinencoding genes, which is consistent with previous reports (Qiu et al., 2016). These two conserved and stress-responsive miRNA families were chosen for phylogenetic analysis not only to validate conservation between the newly identified miRNA members and previously discovered members in the same miRNA family, but also to determine the phylogeny of the stress-responsive miRNAs in this highly adaptive tree.

The miRNAs of angiosperm and gymnosperms always form a separate cluster, indicating the presence of deep evolutionary conservation inside of these two miRNA families. For example, miR166 is closely related to gymnosperms species ( $P$. taeda and $P$. densata), and the precursor sequence of por-miR482a has shown similar evolutionary relationship with other gymnosperms species of $P$. taeda, and P. densata (Fig. 1). However, the homologs across different lineages of plants were also identified (e.g., por-miR482a-3p and zma-miR482; vvi-166d and por-miR166a-5p/3p), which further indicates that the ancient 


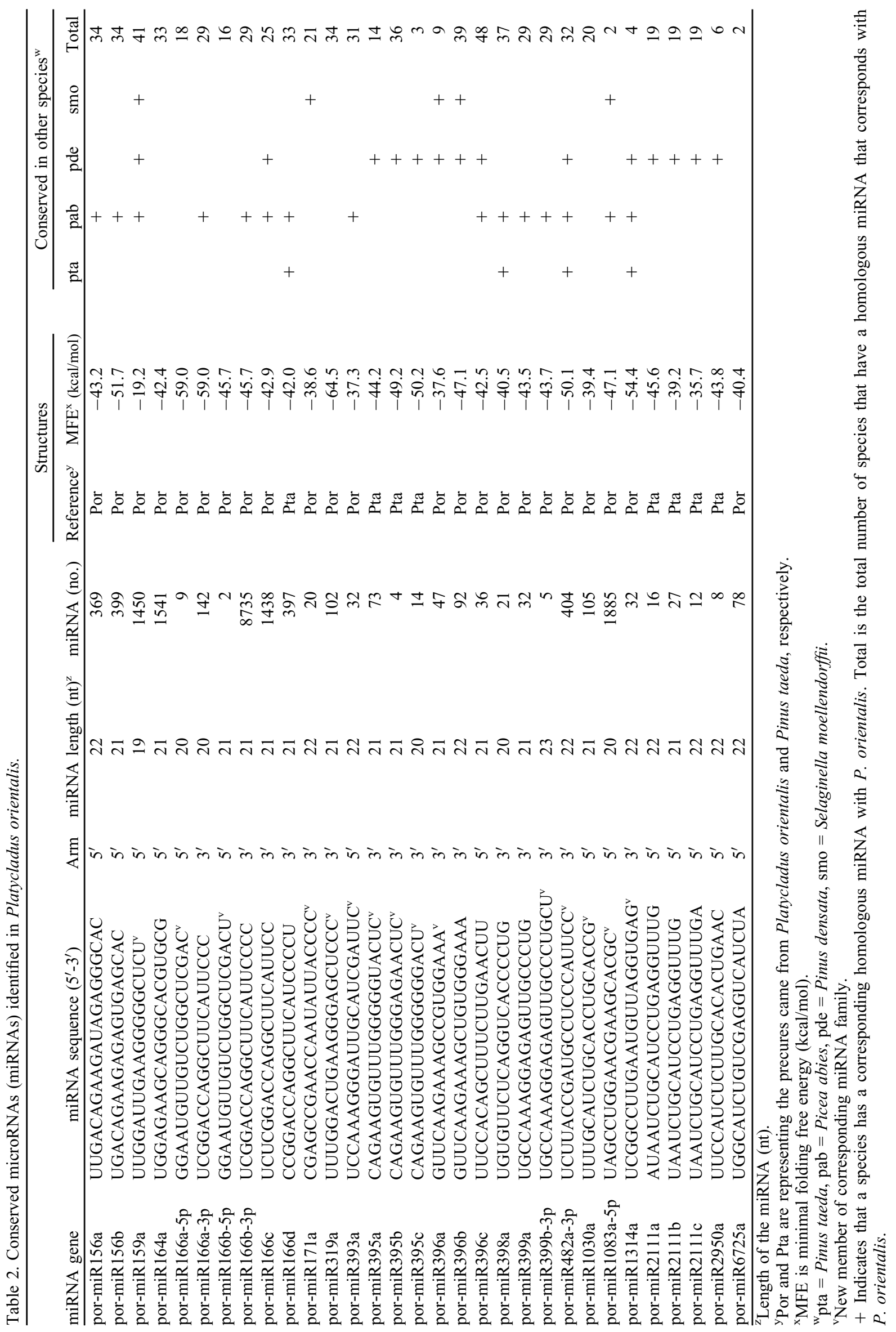




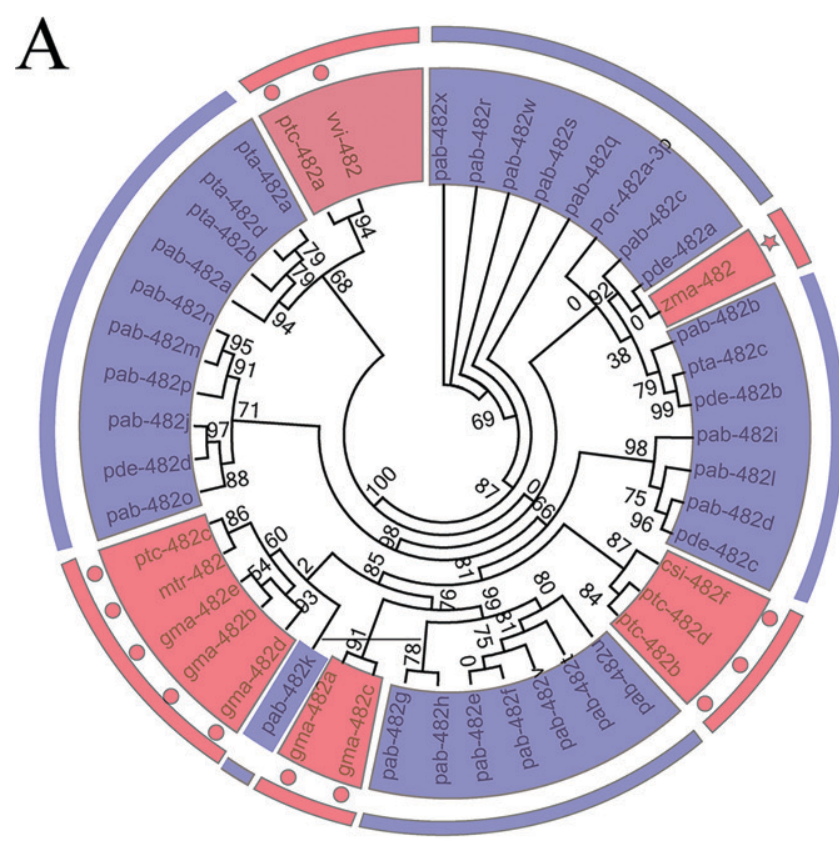

Gymnosperms
Angiosperm
Ferns
$\quad$ Dicotyledons
pde-Pinus densata
pta-Pinus taeda
pab-Piceaabies
ptc-Populus trichocarpa
smo-Selaginella moellendorffii
sbi-Sorghum bicolor
gma-Glycine max
mtr-Medicago truncatula
bna-Brassica napus
ath-Arabidopsis thaliana
osa-Oryza sativa
zma-Zea mays
atr-Amborella trichopoda
csi-Citrus sinensis
vvi-Vitis vinifera

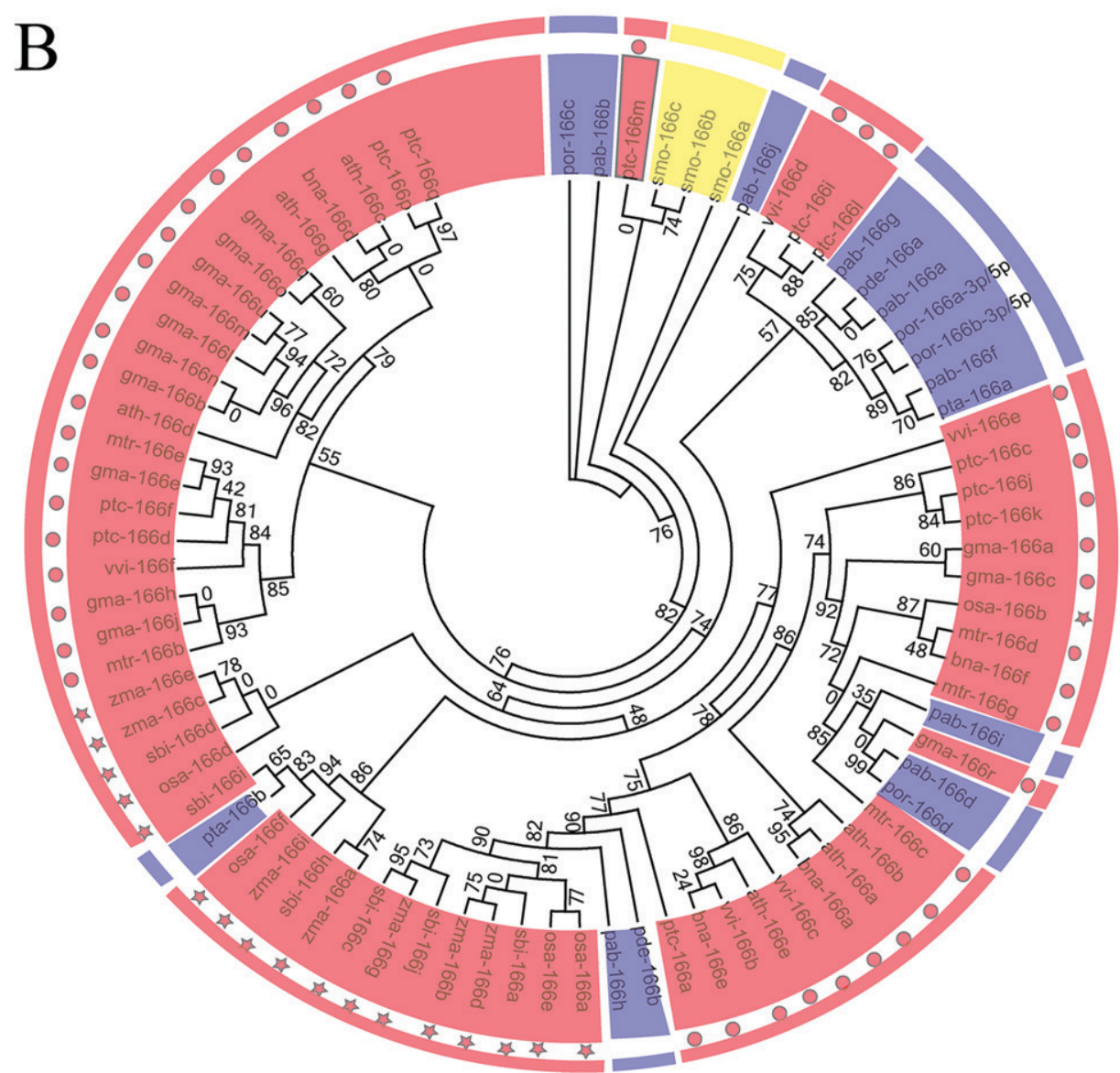

Fig. 1. A neighbor-joining phylogenetic tree of pre-miR482 (A) and pre-miR166 sequences (B) in dicot, monocot, gymnosperm, and lycophyte plants.

miRNA regulatory system is well developed in the common ancestors of gymnosperms and angiosperms (Axtell, 2008).

IDENTIFICATION OF NOVEL MIRNAS OF P. ORIENTALIS. On the basis of the criteria developed by Meyers et al. (2008; see
Methods), 91 sRNAs were identified as novel miRNAs, comprising 77 families (Supplemental Table 3). The precursors of these potential novel miRNAs were formed by a proper secondary hairpin structure with MFE ranging from -18.4 to $-59.8 \mathrm{kcal} / \mathrm{mol}$, 
Table 3. Orthologs of putative novel Platycladus orientalis microRNAs (miRNAs) conserved in other species.

\begin{tabular}{|c|c|c|c|c|c|c|c|c|c|}
\hline \multirow[b]{2}{*}{ miRNA } & \multirow[b]{2}{*}{ sequence $\left(5^{\prime}-3^{\prime}\right)$} & \multirow[b]{2}{*}{ Len. $^{\mathrm{z}}$} & \multicolumn{2}{|c|}{ Highest homology } & \multicolumn{5}{|c|}{ Conserved in other plants ${ }^{\mathrm{w}}$} \\
\hline & & & $\mathrm{Name}^{\mathrm{y}}$ & $\begin{array}{l}\text { Mismatched } \\
\text { bases (no.) }^{\mathrm{x}}\end{array}$ & pta & $\mathrm{pab}$ & pde & smo & total \\
\hline por-miR02 & GGGAUGAAUCUGAGAACACAUG & 22 & gma-miR398b-5p & 7 & & & & & 4 \\
\hline por-miR08 & CGCUGACAUUGGAGGCCGACG & 21 & pab-miR1314 & 4 & & + & + & & 2 \\
\hline por-miR10 & CGUAAUGUUGGUUCGGUUCAGC & 22 & ppe-miR171d-5p & 5 & & & & & 28 \\
\hline por-miR37 & UUAGGGUUAGGAAGAAGAAAUAAU & 24 & ath-miR399b & 3 & & + & & & 29 \\
\hline por-miR61b-3p & UUUUUCCUGCACCACCCAUUCC & 22 & ppe-miR482f & 3 & & + & + & & 20 \\
\hline por-miR61a-3p & UUUUCCCUGAACCACCCAUUCC & 22 & mes-miR482 & 4 & & + & + & & 11 \\
\hline por-miR62a & UUUUCUCUGUACCACCCAUUCC & 22 & vvi-miR3633a-3p & 7 & & + & + & & 8 \\
\hline por-miR63b-5p & CAUGGGAGGGUUGGCAAAAUU & 21 & ppe-miR482b-5p & 6 & & & & & 6 \\
\hline por-miR63b-3p & UCUUGCCAGUCCCUCCAAUGCC & 21 & pgi-miR482a & 4 & & + & & & 12 \\
\hline por-miR63c-5p & CUUCGGGGGAAAGGAAAAAUU & 21 & osa-miR2118 & 5 & + & + & + & & 8 \\
\hline por-miR63c-3p & UUUUCCCGAUUCCUCCGAAGCC & 21 & osa-miR2118 & 5 & + & + & + & & 8 \\
\hline por-miR64b-3p & UCUUACCCGACCCUCCCAUGCC & 21 & csi-miR482b-3p & 4 & & + & & & 12 \\
\hline por-miR68-5p & CCUCUCCCUCAAAGGUUUCC & 20 & ppt-miR477c & 4 & & & & & 18 \\
\hline por-miR399b-5p & CAGGGGAACGCUCCUUUAGCAGG & 23 & ath-miR399b & 3 & & + & & & 29 \\
\hline por-miR1083a-5p & GUGUUUCGUUUCAGGCUUAUG & 22 & smo-miR1083 & 7 & & + & & + & 2 \\
\hline
\end{tabular}

${ }^{\mathrm{z}}$ Length of the miRNA (nucleotides).

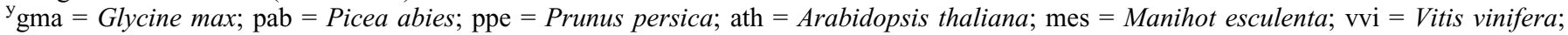
pgi = Panax ginseng; osa $=$ Oryza sativa $;$ csi $=$ Citrus sinensis $;$ ppt $=$ Physcomitrella patens $;$ smo = Selaginella moellendorffi .

${ }^{\mathrm{x}}$ mis. $=$ the number of mismatch base.

${ }^{\mathrm{w}} \mathrm{pta}=$ Pinus taeda; $\mathrm{pde}=$ Pinus densata.

+ Indicates that a species has a corresponding homologous miRNA with $P$. orientalis. Total is the total number of species that have a homologous miRNA that corresponds with $P$. orientalis.

with an average of $-34.8 \mathrm{kcal} / \mathrm{mol}$ (Supplemental Fig. 3). The expression abundances of the novel miRNAs are from 3 to 3936, with an average of 131; however, the expression levels of most $(83 \%)$ of these novel miRNAs are far below this average value. Interestingly, eight (11\%) novel miRNA families have more than one member (e.g., por-MIR64 family has five members, which is the largest family in novel miRNAs). Most importantly, $15 \mathrm{miR}$ NAs were predicted with an antisense miRNA (miRNA*), including three conserved miRNAs (por-miR399b-3p, -miR482a-3p, and -miR1083a-5p) (Supplemental Fig. 4).

Among the novel miRNAs of $P$. orientalis, $73(80 \%)$ and 18 (20\%) miRNA's precursor sequences came from $P$. orientalis and P. taeda, respectively (Supplemental Table 3). To determine whether the novel miRNAs were conserved in other plants, all of the 91 novel miRNAs were used as query sequences and BLASTN searched at miRBase database. Interestingly, 27 novel $(29 \%)$ miRNAs were found have homologs to other plant species, and all of their precursor sequences came from $P$. orientalis. However, of these, only 15 novel $(16 \%)$ miRNAs have homologs with more than two species (mismatch bases between 3 and 7) (Table 3). On the other hand, homology was not found for the remaining 66 novel miRNAs, and this may indicate that they are $P$. orientalis-specific.

VALIDATION OF P. ORIENTALIS MIRNA. All the randomly selected 10 miRNAs of $P$. orientalis could be readily detected by qRTPCR (Fig. 2), suggesting that they are bona fide miRNAs of $P$. orientalis. However, their expression level varied significantly, the average $\Delta \mathrm{Ct}$ of the selected novel miRNAs was 0.69 , which was much lower than conserved miRNAs (the average $\Delta \mathrm{Ct}=13.99$ ). All the selected conserved miRNAs appeared to be highly expressed, suggesting their conservative and primary function in essential biological processes of $P$. orientalis. Given the random nature of miRNA selection, only two novel miRNAs of $P$. orientalis (por-miRNA55 and -miRNA67-5p) were selected, but all appeared to be expressed at lower levels compared with other conserved miRNAs. This is consistent with previously reported studies indicating that the relative expression levels of nonconserved miRNAs are usually at lower levels with tissue or developmental phase-specific patterns (Ruan et al., 2009; Song et al., 2010). Our validated results suggest that RNA sequencing was capable of successfully discovering candidate miRNAs from $P$. orientalis with high accuracy and efficiency.

IDENTIFICATION AND ANNOTATION OF MIRNA TARGETS. A total of 148,867 sequences from $P$. orientalis mRNA transcriptome database and 122 identified miRNAs in this study were used as custom target and custom miRNA databases in the web-based program psRNATarget. Finally, 2331 unique mRNAs were predicted to be targets of the 122 miRNAs (Supplemental Table 4), suggesting that most of the miRNAs were predicted to have more than one target and that one target could be regulated by several miRNAs. These target annotations show that $56.6 \%$ can be annotated in the $\mathrm{Nr}$ database, and they were involved in a

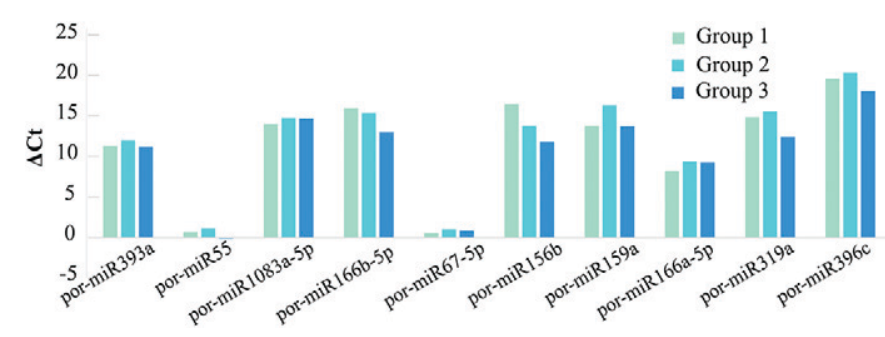

Fig. 2. Quantitative real-time polymerase chain reaction validation of the identified miRNAs in Platycladus orientalis. The $\Delta \mathrm{Ct}$ value means the relative expression level of the verified miRNA; Groups 1, 2, and 3 represent three independent biological replicates. 
variety of physiological processes in plants (Supplemental Table 4). Approximately $11(36 \%)$ conserved miRNA targets were homologous to previously validated or predicted miRNA targets in Arabidopsis thaliana (Hsieh et al., 2009; Liu et al., 2008, 2009), Oryza sativa (Zhou et al., 2010), Prunus persica (Eldem et al., 2012), Populus trichocarp (Shuai et al., 2013), Medicago truncatula (Wang et al., 2011), P. densata (Wan et al., 2012), Triticum dicoccoides (Kantar et al., 2011), Gossypium raimondii (Zhu et al., 2013), and Glycine max (Li et al., 2011; Xu et al., 2013) (Table 4). Additionally, most of these homology targets are plant-specific transcription factors and involved in stem and leaf development, flower and seed formation, root development, and the coordination of growth processes under adverse environmental conditions.

GO CLASSIFICATION AND KEGG PATHWAY ANALYSIS OF THE PREDICTED MIRNA TARGETS. In the present study, $\approx 39.2 \%$ targets could be annotated in GO database and categorized in terms of molecular functions (118 miRNAs), biological processes (117), and cellular components (112) (Supplemental Table 5). The GO enrichment analysis further disclosed that $P$. orientalis miRNAs was primarily involved with ATP binding (81), the nucleus (69), and the oxidation-reduction process (67) in categories of molecular functions, cellular components, and biological processes, respectively (Fig. 3). Interestingly, 27 and 22 miRNAs are significantly enriched in defense and stress responses, respectively.
Pathway-based analysis of the predicted miRNA targets shows that $P$. orientalis miRNAs are involved in 187 KEGG pathways (Supplemental Table 6), which could be assigned to five main categories of cellular processes (involved 19 miRNAs), environmental information processing (4), genetic information processing (27), metabolism (68), and organismal systems (28). We found that one miRNA may take part in several pathways and that one pathway could be regulated by several miRNAs. A more detailed distribution of miRNA in the subcategories is shown in Fig. 4, of which the carbohydrate metabolism is the subcategory that has been regulated most frequently by miRNAs, followed by global and overview maps (28), lipid metabolism (26), amino acid metabolism (25), signal transduction (23), and other functions.

GO annotation shows involvement of a number of miRNAs in response to different biotic and abiotic stresses. For example, GO enrichment found 20 miRNAs of $P$. orientalis (por-miR16, -miR22, -miR28, -miR32, -miR33, -miR35, -miR38, -miR39, -miR42, -miR57b, -miR59b, -miR60-3p, -miR60-5p, -miR61b-3p, -miR63c-5p, -miR67-5p, -miR396c, -miR396b, -miR1083a-5p, -miR6725a) involved in response to stress. Pathway-based analysis displayed a deeper insight into the associated metabolic and biosynthetic pathways with miRNA, such as por-miR396b play an important role in the pathway of plant hormone signal transduction (KEGG ID: ko04075) and -miR33 have a function in pathways of proteasome (ko03050), alpha-Linolenic acid

Table 4. Conserved Platycladus orientalis microRNA (miRNA) targets and their putative functions.

\begin{tabular}{|c|c|c|c|c|}
\hline Target function $^{\mathrm{z}}$ & miRNA & Target $^{\mathrm{y}}$ & Species $^{x}$ & Source \\
\hline $\begin{array}{l}\mathrm{MYB}^{\mathrm{z}} \text { transcription factors- } \\
\mathrm{ABA}^{\mathrm{z}} \text { response, } \mathrm{NaCl} \text { stress } \\
\text { response, floral }\end{array}$ & por-miR159a & comp61508_c0_seq2 (2.5) & Ath, Osa, Ppe & $\begin{array}{l}\text { Eldem et al., 2012; } \\
\text { Liu et al., 2008; } \\
\text { Zhou et al., } 2010\end{array}$ \\
\hline $\begin{array}{l}\text { HD-ZIPIII transcription factor- } \\
\text { axillary meristem initiation, } \\
\text { leaf and vascular } \\
\text { development }\end{array}$ & $\begin{array}{l}\text { por-miR166a-3p } \\
\text { por-miR166b-3p } \\
\text { por-miR166d }\end{array}$ & $\begin{array}{l}\text { comp53851_c0_seq2 (1) } \\
\text { comp58082_c0_seq1 (1) } \\
\text { comp53851_c0_seq2 (1) } \\
\text { comp58082_c0_seq1 (1) } \\
\text { comp53851_c0_seq2 (0) } \\
\text { comp58082_c0_seq1 (0) }\end{array}$ & Ptc, Tdi, Gma & $\begin{array}{l}\text { Kantar et al., 2011; } \\
\text { Li et al., 2011; } \\
\text { Shuai et al., } 2013\end{array}$ \\
\hline $\begin{array}{l}\text { TIR1 and AFB2 and AFB3- } \\
\text { susceptibility to virulent } \\
\text { bacteria }\end{array}$ & por-miR393a & comp24630_c0_seq1 (1.5) & Ath, Ppe & $\begin{array}{l}\text { Eldem et al., 2012; } \\
\text { Liu et al., } 2008\end{array}$ \\
\hline $\begin{array}{l}\text { Growth-regulating factor-stem, } \\
\text { leaf development, growth } \\
\text { processes }\end{array}$ & por-miR396a & $\begin{array}{l}\text { comp46995_c0_seq1 (3) } \\
\text { comp60601_c0_seq3 (3) }\end{array}$ & Ath & Liu et al., 2008, 2009 \\
\hline $\begin{array}{l}\text { Kelch repeat-containing } \\
\text { F-box protein-regulation } \\
\text { of mineral homeostasis }\end{array}$ & $\begin{array}{l}\text { por-miR2111a } \\
\text { por-miR2111b } \\
\text { por-miR2111c }\end{array}$ & $\begin{array}{l}\text { comp72982_c0_seq1 }(0.5) \\
\text { comp72982_c0_seq1 }(0.5) \\
\text { comp72982_c0_seq1 }(0.5)\end{array}$ & Ath, Gma & $\begin{array}{l}\text { Hsieh et al., 2009; } \\
\text { Xu et al., } 2013\end{array}$ \\
\hline
\end{tabular}




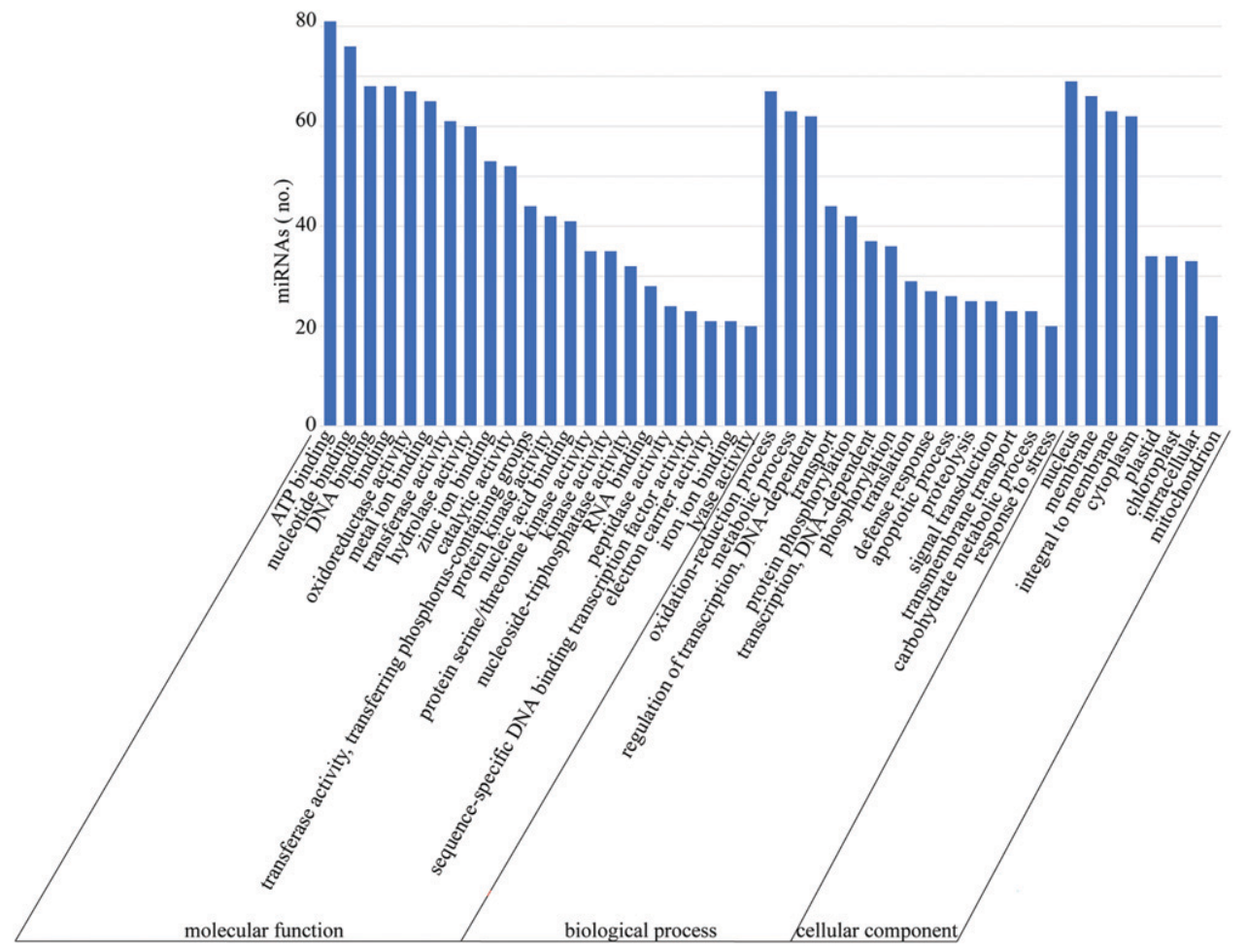

Fig. 3. Gene ontology term enrichment analysis of the predicted targets. miRNAs = microRNAs.

metabolism (ko00592), and protein digestion and absorption (ko04974). Additionally, por-miR16, -miR44, -miR60-5p, -miR69-3p, -miR166b-5p, and por-miR395c also were identified in environmental adaptation pathway by KEGG annotation.

\section{Discussion}

The sRNA-mediated transcriptional or posttranscriptional regulation of gene expression, including the miRNA and siRNA

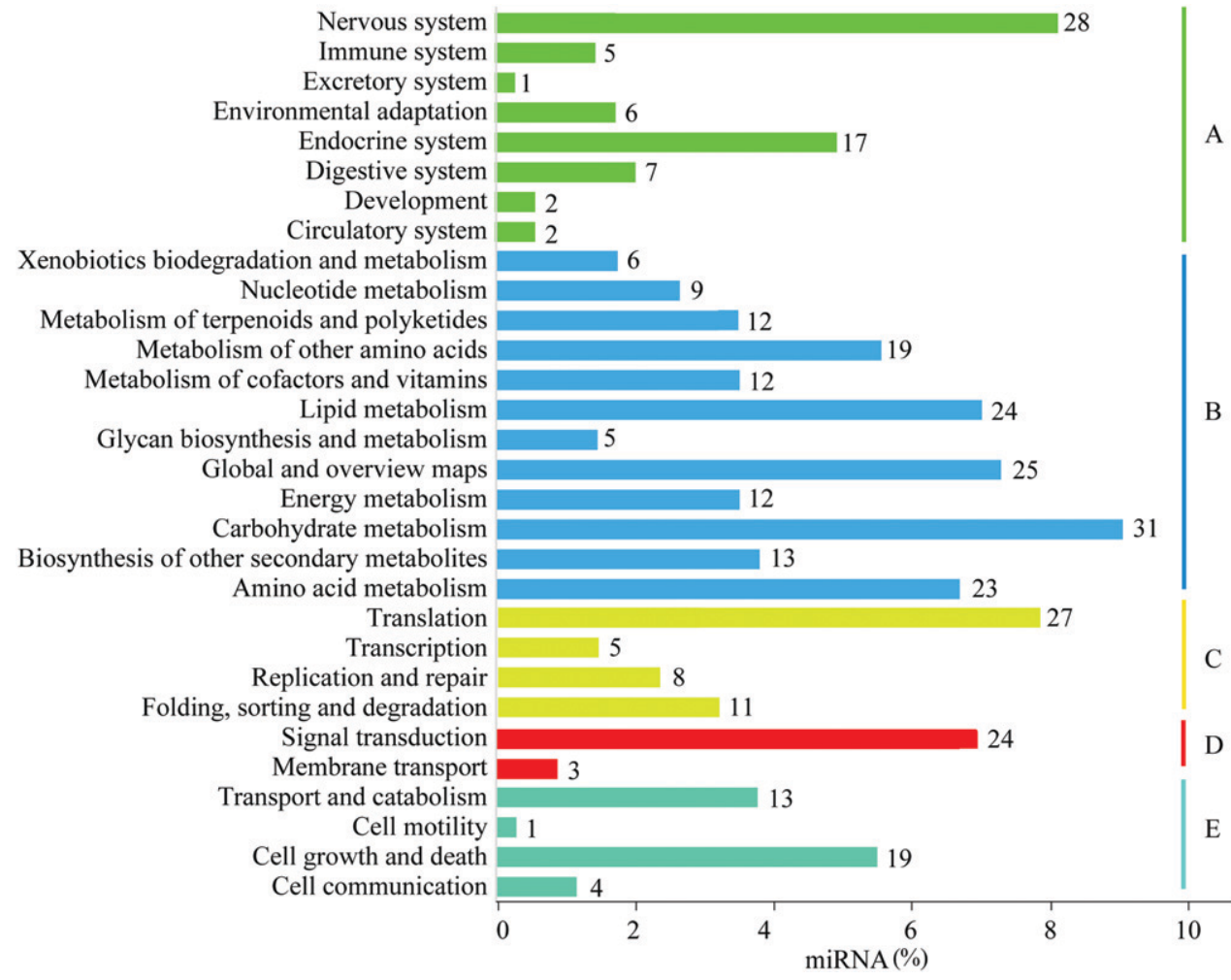

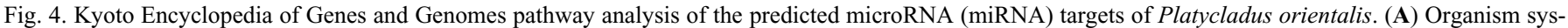
tem, (B) metabolism, (C) genetic information processing, (D) environmental information processing, and (E) cellular processes. 
pathways, which is an important epigenetic regulatory mechanism in plants. In this study, with the deep sequencing, a total of $1,314,450$ unique reads were obtained from a stress-tolerant conifer, $P$. orientalis, and 31 conserved and 91 novel miRNAs were identified and characterized. Targets predicted and annotated shows that $P$. orientalis miRNAs regulate gene expression post-transcriptionally and act in a wide range of biological and metabolic processes, which is particularly useful for identifying specific miRNAs and pathways that related to adaptive mechanism of this stress-tolerant species.

The length of the identified mature and pre-miRNAs varied within a reasonable scope, which is in agreement with previous reports on gymnosperm, such as $P$. glauca (Liu and El-Kassaby, 2017), P. balfouriana (Li et al., 2017), and P. densata (Wan et al., 2012), and angiosperm plantsthat is, A. thaliana (Rajagopalan et al., 2006) and $P$. trichocarpa (Klevebring et al., 2009). The thermodynamical characteristic MFE is an important criterion for distinguishing miRNAs from other noncoding RNAs. The RNA secondary structure with lower MFE is generally more stable than those with higher MFE (Bonnet et al., 2004; Li and Zhang, 2011). The predicted $P$. orientalis pre-miRNAs have a low MFE with an average of $-43.90 \mathrm{kcal} / \mathrm{mol}$ (Table 2, Supplemental Table 2). It is similar to the miRNAs in other plant species, such as $P$. densata and $M$. truncatula (Wan et al., 2012; Wang et al., 2011). Additionally, although most of conserved miRNAs of $P$. orientalis could be found in other plant species, the conservative is not always in conifers and other gymnosperms (i.e., por-miR164 is the first report in gymnosperms).

A phylogenetic study was useful to advance our insight into the evolutionary relationship of $P$. orientalis miRNAs with other plant species. The precursor sequences showed a high degree of similarity between the new identified member and other alreadyreported members in families of miR166 and miR482. In addition, it is clear that the precursor sequence of $P$. orientalis miRNAs could be clustered with other gymnosperms [i.e., Picea abies (pab), P. taeda (pta), P. densata (pde), Selaginella moellendorffii (smo)] in MIR166 and MIR482 phylogenetic trees, respectively (Fig. 1). This was consistent with previous reports that members within the same family are highly conserved among various species (Taylor et al., 2014).

The miRNA* is an incomplete complementarity singlestranded RNA of $\approx 22$ nucleotide lengths and is produced in the process of synthesizing mature miRNAs. Previous studies have shown that the expression abundance of miRNAs* is much lower than the corresponding miRNAs (Berezikov et al., 2011; Blahna and Hata, 2013). In addition, the stability of the miRNA* $5^{\prime}$ end is better than that of the $3^{\prime}$ end of miRNA; this thermodynamic asymmetry makes miRNA more likely to be selected in the RNA-induced silencing complex, and the complementary miRNAs* are more likely to be degraded; therefore, miRNAs* have been considered ineffective in previous studies (Berezikov et al., 2011; Meng et al., 2011). However, with the in-depth research on miRNAs, several studies have found that miRNAs* are involved in the posttranscriptional regulatory network as miRNA. Recent studies have shown that the expression abundance of miRNA* is not lower than that of corresponding miRNAs in some biological tissues or is even much higher than miRNAs (Choo et al., 2014; Yonemori et al., 2016). In our study, there are also no certain expression patterns of the sense and antisense miRNAs in $P$. orientalis. For example, the expression abundances were 3936 and 19 for por-miR61a-3p/5p, respectively, showing significant differences, but there seem to be no significant differences in por-miR68-3p/5p, with expression abundances of 14 and 18 , respectively.

In this study, the transcription factor of the NAC domain and HD-ZIPIII were predicted to be targets of por-MIR164 and -MIR166, respectively, and these transcription factors were validated as playing important roles in root, leaf, vascular, and nodule development in M. truncatula, $P$. trichocarpa, and T. dicoccoides (Kantar et al., 2011; Shuai et al., 2013; Wang et al., 2011). Furthermore, NBS-LRR resistance-like protein, an important nontranscriptional factor protein, plays a critical regulatory role in disease resistance in plants (Eldem et al., 2012; Liu et al., 2008; Zhou et al., 2010); it also was predicted to be regulated by porMIR482 in this study. These results show that $P$. orientalis miRNA targets encoded not only indispensable transcription factors but also nontranscriptional factor proteins involved in diverse physiological processes. Interestingly, the miRNA families MIR164, MIR166, MIR393, and MIR159 have been reported to be involved in physiological and biochemical metabolism of drought response in various species (Shuai et al., 2013; Wang et al., 2011), and these drought-related miRNAs were also found in $P$. orientalis, suggesting that $P$. orientalis may have its own defense systems to cope with adverse climatic conditions, and miRNA may play an important role in these metabolic systems.

A total of $187 \mathrm{KEGG}$ pathways in $P$. orientalis were found to be regulated by miRNAs, providing a direction for studying the specific role of $P$. orientalis miRNAs, and especially for understanding the physiological and genetic mechanisms of the specie's extraordinary adaptability to adverse environmental conditions. For example, six miRNAs targets were found to be involved in environmental adaptation pathways, including por-miR69-3p in circadian entrainment; por-miR395c in circadian rhythm; and pormiR16, -miR60-5p, -miR44, and -miR166b-5p in plant-pathogen interaction. Climate change has caused frequent drought stress at the flowering stage of $P$. orientalis in the northern region of China, which may result in declines of pollen fertility in $P$. orientalis. The flowering stage of $P$. orientalis is a period sensitive to environmental pressure, and therefore, the identified miRNAs related to adaptation are significant discoveries for regulating internal metabolism activity and improving adaptability. Additionally, pathway-based analysis could further our understanding of the biological functions and interactions of miRNAs, because the functional annotation results assigned to the same pathway may mean that these miRNAs were functionally related with each other. Therefore, our study opens a new avenue for studying specific miRNAs and pathways in this species, and have potential value in assisted breeding and conservation applications.

\section{Conclusions}

In this study, 31 conserved and 91 novel miRNAs in $P$. orientalis were identified and analyzed. Of these, 15 conservative miRNAs were discovered first; 15 miRNAs were found with an antisense miRNA; and the conservatism of newly identified and previously discovered members were detected by phylogenetic analysis in MIR166 and MIR482, respectively. Additionally, the accuracy and reproducibility of the identified $P$. orientalis miRNAs were validated by qRT-PCR. Target prediction and annotation showed that $P$. orientalis miRNAs involved in a wide range of biological and metabolic processes, which is particularly useful for understanding specific miRNAs and pathways 
that are related to adaptive mechanisms of this highly adaptable species.

\section{Literature Cited}

Altschul, S.F., W. Gish, W. Miller, E.W. Myers, and D.J. Lipman. 1990. Basic local alignment search tool. J. Mol. Biol. 215:403-410, https://doi.org/10.1002/9780470451496.ch4.

Axtell, M.J. 2008. Evolution of microRNAs and their targets: Are all microRNAs biologically relevant? Biochim. Biophys. Acta. Gene Regul. Mech. 1779:725-734, https://doi.org/10.1016/j.bbagrm.2008. 02.007.

Berezikov, E., N. Robine, A. Samsonova, J.O. Westholm, A. Naqvi, J.-H. Hung, K. Okamura, Q. Dai, D. Bortolamiol-Becet, and R. Martin. 2011. Deep annotation of Drosophila melanogaster microRNAs yields insights into their processing, modification, and emergence. Genome Res. 21:203-215, https://doi.org/10.1101/gr.116657.110.

Blahna, M.T. and A. Hata. 2013. Regulation of miRNA biogenesis as an integrated component of growth factor signaling. Curr. Opin. Cell Biol. 25:233-240, https://doi.org/10.1016/j.ceb.2012.12.005.

Bonnet, E., J. Wuyts, P. Rouzé, and Y. Van de Peer. 2004. Evidence that microRNA precursors, unlike other non-coding RNAs, have lower folding free energies than random sequences. Bioinformatics 20:2911-2917, https://doi.org/10.1093/bioinformatics/bth374.

Bustin, S.A., V. Benes, J.A. Garson, J. Hellemans, J. Huggett, M. Kubista, R. Mueller, T. Nolan, M.W. Pfaffl, and G.L. Shipley. 2009. The MIQE guidelines: Minimum information for publication of quantitative real-time PCR experiments. Clin. Chem. 55:611-622, https://doi.org/10.1373/clinchem.2008.112797.

Chen, C.Z., S. Schaffert, R. Fragoso, and C. Loh. 2013. Regulation of immune responses and tolerance: The micro RNA perspective. Immunol. Rev. 253:112-128, https://doi.org/10.1111/imr.12060.

Choo, K.B., Y.L. Soon, P.N.N. Nguyen, M.S.Y. Hiew, and C.-J. Huang. 2014. MicroRNA-5p and-3p co-expression and cross-targeting in colon cancer cells. J. Biomed. Sci. 21:95, https://doi.org/10.1186/s12929014-0095-x.

Conesa, A., S. Götz, J.M. García-Gómez, J. Terol, M. Talón, and M. Robles. 2005. Blast2GO: A universal tool for annotation, visualization and analysis in functional genomics research. Bioinformatics 21:3674-3676, https://doi.org/10.1093/bioinformatics/bti610.

Dai, X., Z. Zhuang, and P.X. Zhao. 2018. psRNATarget: A plant small RNA target analysis server (2017 release). Nucleic Acids Res. 46:49-54, https://doi.org/10.1093/nar/gky316.

Dong, T.M., X.Y. Chen, X.M. Zhang, Z.R. Li, and W.H. Kong. 1990. Platycladus orientalis. Henan Sci. Technol. Press, Zhengzhou, China.

Eldem, V., U.C. Akcay, E. Ozhuner, Y. Bakõr, S. Uranbey, and T. Unver. 2012. Genome-wide identification of miRNAs responsive to drought in peach (Prunus persica) by high-throughput deep sequencing. PLoS One 7:e50298, https://doi.org/10.1371/journal.pone.0050298.

Fendler, A., C. Stephan, G.M. Yousef, and K. Jung. 2011. MicroRNAs as regulators of signal transduction in urological tumors. Clin. Chem. Lab. Med. 57:954-968, https://doi.org/10.1373/clinchem.2010.157727.

Ferdous, J., S.S. Hussain, and B.J. Shi. 2015. Role of microRNA s in plant drought tolerance. Plant Biotechnol. J. 13:293-305, https://doi. org/10.1111/pbi.12318.

Flora of China Editorial Committee. 1978. Flora Reipublicae Popularis Sinicae. Beijing. Science Press 7:321-322.

Griffiths-Jones, S., H.K. Saini, D.S. Van, and A.J. Enright. 2008. miRBase: Tools for microRNA genomics. Nucleic Acids Res. 36: 154-158, https://doi.org/10.1093/nar/gkm952.

Hsieh, L.-C., S.-I. Lin, A.C.-C. Shih, J.-W. Chen, W.-Y. Lin, C.-Y. Tseng, W.-H. Li, and T.-J. Chiou. 2009. Uncovering small RNAmediated responses to phosphate deficiency in Arabidopsis by deep sequencing. Plant Physiol. 151:2120-2132, https://doi.org/10.1104/ pp.109.147280.

Hu, X.-G., Y. Jin, X.-R. Wang, J.-F. Mao, and Y. Li. 2015. Predicting impacts of future climate change on the distribution of the widespread conifer Platycladus orientalis. PLoS One 10:e0132326, https://doi.org/10.1371/journal.pone.0132326.

Hu, X.-G., H. Liu, Y. Jin, Y.-Q. Sun, Y. Li, W. Zhao, Y.A. ElKassaby, X.-R. Wang, and J.-F. Mao. 2016. De novo transcriptome assembly and characterization for the widespread and stress-tolerant conifer Platycladus orientalis. PLoS One 11:e0148985, https://doi. org/10.1371/journal.pone.0148985.

Hu, X.-G., T. Wang, S.-S. Liu, S.-Q. Jiao, K.-H. Jia, S.-S. Zhou, Y. Jin, Y. Li, Y.A. El-Kassaby, and J.-F. Mao. 2017. Predicting future seed sourcing of Platycladus orientalis (L.) for future climates using climate niche models. Forests 8:471, https://doi.org/10.3390/f8120471.

Kantar, M., S.J. Lucas, and H. Budak. 2011. miRNA expression patterns of Triticum dicoccoides in response to shock drought stress. Planta 233:471-484, https://doi.org/10.1007/s00425-010-1309-4.

Klevebring, D., N.R. Street, N. Fahlgren, K.D. Kasschau, J.C. Carrington, J. Lundeberg, and S. Jansson. 2009. Genome-wide profiling of populus small RNAs. BMC Genomics 10:620, https://oi.org/10.1186/14712164-10-620.

Kozomara, A., M. Birgaoanu, and S. Griffiths-Jones. 2018. miRBase: From microRNA sequences to function. Nucleic Acids Res. 47:D155-D162, https://doi.org/10.1093/nar/gky1141.

Lagos-Quintana, M., R. Rauhut, W. Lendeckel, and T. Tuschl. 2001. Identification of novel genes coding for small expressed RNAs. Science 294:853-858, https://doi.org/10.1126/science.1064921.

Larkin, M.A., G. Blackshields, N.P. Brown, R. Chenna, P.A. McGettigan, H. McWilliam, F. Valentin, I.M. Wallace, A. Wilm, R. Lopez, J.D. Thompson, T.J. Gibson, and D.G. Higgins. 2007. ClustalW and ClustalX version 2.0. Bioinformatics 23:2947-2948, https://doi.org/10.1093/ bioinformatics/btm 404 .

Li, H., Y. Dong, H. Yin, N. Wang, J. Yang, X. Liu, Y. Wang, J. Wu, and X. Li. 2011. Characterization of the stress associated microRNAs in Glycine max by deep sequencing. BMC Plant Biol. 11:170, https://doi.org/10.1186/1471-2229-11-170.

Li, Q., D. Cheng, X. Yan, L. Kong, H. Zhang, S. Zhang, and J. Wang. 2017. Identification of novel miRNAs and miRNA expression profiling in embryogenic tissues of Picea balfouriana treated by 6-benzylaminopurine. PLoS One 12:e176112, https://doi.org/10.1371/journal. pone.0176112.

Li, Y. and S. Zhang. 2011. Finding stable local optimal RNA secondary structures. Bioinformatics 27:2994-3001, https://doi.org/10.1093/ bioinformatics/btr510.

Li, Y., Z. Zhang, F. Liu, W. Vongsangnak, Q. Jing, and B. Shen. 2012. Performance comparison and evaluation of software tools for microRNA deep-sequencing data analysis. Nucleic Acids Res. 40:4298-4305, https://doi.org/10.1093/nar/gks043.

Liu, D., Y. Song, Z. Chen, and D. Yu. 2009. Ectopic expression of miR396 suppresses GRF target gene expression and alters leaf growth in Arabidopsis. Physiol. Plant. 136:223-236, https://doi.org/ 10.1111/j.1399-3054.2009.01229.x.

Liu, H.-H., X. Tian, Y.-J. Li, C.-A. Wu, and C.-C. Zheng. 2008. Microarray-based analysis of stress-regulated microRNAs in Arabidopsis thaliana. RNA 14:836-843, https://doi.org/10.1261/rna.895308.

Liu, Y. and Y.A. El-Kassaby. 2017. Landscape of fluid sets of hairpinDerived 21-/24-nt-long small RNAs at seed set uncovers special epigenetic features in Picea glauca. Genome Biol. Evol. 9:82-92, https://doi.org/10.1093/gbe/evw283.

Livak, K.J. and T.D. Schmittgen. 2001. Analysis of relative gene expression data using real-time quantitative PCR and the $2^{-\Delta \Delta C T}$ method. Methods 25:402-408, https://doi.org/10.1006/meth.2001.1262.

Meng, Y., C. Shao, L. Gou, Y. Jin, and M. Chen. 2011. Construction of microRNA-and microRNA*-mediated regulatory networks in plants. RNA Biol. 8:1124-1148, https://doi.org/10.4161/rna.8.6.17743.

Meyers, B.C., M.J. Axtell, B. Bartel, D.P. Bartel, D. Baulcombe, J.L. Bowman, X. Cao, J.C. Carrington, X. Chen, and P.J. Green. 2008. Criteria for annotation of plant MicroRNAs. Plant Cell 20:3186-3190, https://doi.org/10.1105/tpc.108.064311. 
Qiu, Z.-B., M.-M. Yuan, B.-Z. Hai, L. Wang, and L. Zhang. 2016. Characterization and expression analysis of conserved miRNAs and their targets in Pinus densata. Biol. Plant. 60:427-434, https://doi. org/10.1007/s10535-016-0617-3.

Rajagopalan, R., H. Vaucheret, J. Trejo, and D.P. Bartel. 2006. A diverse and evolutionarily fluid set of microRNAs in Arabidopsis thaliana. Genes Dev. 20:3407-3425, https://doi.org/10.1101/gad.1476406.

Ruan, M.-B., Y.-T. Zhao, Z.-H. Meng, X.-J. Wang, and W.-C. Yang. 2009. Conserved miRNA analysis in Gossypium hirsutum through small RNA sequencing. Genomics 94:263-268, https://doi.org/ 10.1016/j.ygeno.2009.07.002.

Shuai, P., D. Liang, Z. Zhang, W. Yin, and X. Xia. 2013. Identification of drought-responsive and novel Populus trichocarpa microRNAs by high-throughput sequencing and their targets using degradome analysis. BMC Genomics 14:233, https://doi.org/10.1186/1471-2164-14-233.

Song, C., W. Chen, C. Zhang, N.K. Korir, H. Yu, Z. Ma, and J. Fang. 2010. Deep sequencing discovery of novel and conserved microRNAs in trifoliate orange (Citrus trifoliata). BMC Genomics 11:431, https://doi.org/10.1186/1471-2164-11-431.

Sun, G. 2012. MicroRNAs and their diverse functions in plants. Plant Mol. Biol. 80:17-36, https://doi.org/10.1007/s11103-011-9817-6.

Tamura, K., D. Peterson, N. Peterson, G. Stecher, M. Nei, and S. Kumar. 2011. MEGA5: Molecular evolutionary genetics analysis using maximum likelihood, evolutionary distance, and maximum parsimony methods. Mol. Biol. Evol. 28:2731-2739, https://doi.org/ $10.1093 / \mathrm{molbev} / \mathrm{msr} 121$.

Taylor, R.S., J.E. Tarver, S.J. Hiscock, and P.C. Donoghue. 2014. Evolutionary history of plant microRNAs. Trends Plant Sci. 19:175-182, https://doi.org/10.1016/j.tplants.2013.11.008.

Vienberg, S., J. Geiger, S. Madsen, and L.T. Dalgaard. 2017. MicroRNA s in metabolism. Acta Physiol. (Oxf.) 219:346-361, https://doi. org/10.1111/apha.12681.

Wan, L.-C., H. Zhang, S. Lu, L. Zhang, Z. Qiu, Y. Zhao, Q.-Y. Zeng, and J. Lin. 2012. Transcriptome-wide identification and characterization of
miRNAs from Pinus densata. BMC Genomics 13:132, https://doi.org/ 10.1186/1471-2164-13-132.

Wang, T., L. Chen, M. Zhao, Q. Tian, and W.-H. Zhang. 2011. Identification of drought-responsive microRNAs in Medicago truncatula by genome-wide high-throughput sequencing. BMC Genomics 12:367, https://doi.org/10.1186/1471-2164-12-367.

Xie, F., D.C. Jones, Q. Wang, R. Sun, and B. Zhang. 2015. Small RNA sequencing identifies miRNA roles in ovule and fibre development. Plant Biotechnol. J. 13:355-369, https://doi.org/10.1111/pbi.12296.

Xu, F., Q. Liu, L. Chen, J. Kuang, T. Walk, J. Wang, and H. Liao. 2013. Genome-wide identification of soybean microRNAs and their targets reveals their organ-specificity and responses to phosphate starvation. BMC Genomics 14:66, https://doi.org/10.1186/1471-2164-14-66.

Yonemori, M., N. Seki, H. Yoshino, R. Matsushita, K. Miyamoto, M. Nakagawa, and H. Enokida. 2016. Dual tumor-suppressors miR-139$5 p$ and miR-139-3p targeting matrix metalloprotease 11 in bladder cancer. Cancer Sci. 107:1233-1242, https://doi.org/10.1111/cas.13002.

Zheng, Y., H. Zhang, X. Zhang, D. Feng, X. Luo, C. Zeng, K. Lin, H. Zhou, L. Qu, and P. Zhang. 2012. MiR-100 regulates cell differentiation and survival by targeting RBSP3, a phosphatase-like tumor suppressor in acute myeloid leukemia. Oncogene 31:80-92, https://doi. org/10.1038/onc.2011.208.

Zhou, L., Y. Liu, Z. Liu, D. Kong, M. Duan, and L. Luo. 2010. Genome-wide identification and analysis of drought-responsive microRNAs in Oryza sativa. J. Expt. Bot. 61:4157-4168, https://doi. org/10.1093/jxb/erq237.

Zhu, Q.-H., L. Fan, Y. Liu, H. Xu, D. Llewellyn, and I. Wilson. 2013. miR482 regulation of NBS-LRR defense genes during fungal pathogen infection in cotton. PLoS One 8:e84390, https://doi.org/10.1371/ journal.pone. 0084390 .

Zuker, M. 2003. Mfold web server for nucleic acid folding and hybridization prediction. Nucleic Acids Res. 31:3406-3415, https://doi.org/ 10.1093/nar/gkg595. 